\title{
Overview of Dempster-Shafer and Belief Function Tracking Methods
}

\author{
Erik Blasch \\ Jean Dezert \\ B Pannetier
}

\begin{abstract} with permission.

Over the years, there have been many proposed methods in set-based tracking. One example of set-based methods is the use of Dempster-Shafer (DS) techniques to support belief-function (BF) tracking. In this paper, we overview the issues and concepts that motivated DS methods for simultaneous tracking and classification/identification. DS methods have some attributes, if applied correctly; but there are some pitfalls that need to be carefully avoided such as the redistribution of the mass associated with conflicting measurements. Such comparisons and applications are found in Dezert-Smarandache Theory (DSmT) methods from which the Proportional Conflict Redistribution (PCR5) rule supports a more comprehensive approach towards applying evidential and BF techniques to target tracking. In the paper, we overview two decades of research in the area of BF tracking and conclude with a comparative analysis of Bayesian, Dempster-Shafer, and the PCR5 methods.
\end{abstract}

Originally published as Blasch E., Dezert J., Pannetier B., Overview of Dempster-Shafer and Belief Function Tracking Methods, in Proc. SPIE, Vol. 8745, Baltimore, MD, USA, April 2013, and reprinted

Keywords: Dempster-Shafer, Belief Functions, DSmT, Target Recognition, Classification, \& Identification, Tracking

\section{INTRODUCTION}

Humans and machines are typically trained for specific missions and/or scenarios [1]. One such case is classification of a moving target [2]. To integrate the benefits of human reasoning with machine methods, popular techniques of information fusion [3], target tracking [4], and pattern classification [5] are used.

When the human approaches the target, either the target is moving, the human is moving, or both are moving [6]. Cognition, the act of directing attention to sensory information, can be used by the human to fuse track and identify (ID) information as a perception of a set of moving targets. Dynamic cognitive multitarget-multisensor fusion under uncertainty requires target selection which can be formulated as a belief filtering problem in which sensed target states and identities are represented as current situational beliefs. The objective of the human is to 1) abstract number of tracks from the tracking environment, 2) assess confidence levels from the target classification algorithm, and 3) integrate the information for real-time beliefs of the number and types of targets from a plausible set of targets through an interactive display [7].

Multitarget tracking and ID is a subset of sensor fusion, which includes selecting sensors [8], sensor recognition policies, and tracking algorithms for a given set of mission requirements [9] for situational awareness [10,11]. For example, in a typical tactical aircraft, the onboard sensors are active radar, electro-optical/infrared (EO/IR), and navigation sensors, with each sensor having a variety of modes in which it can operate and features it can detect. Figure 1 shows the case of EO/IR targets. The EO/IR sensor makes kinematic measurements to detect, track, and classify objects of interest while reducing user workload. In a dynamic and uncertain environment, a sensor manager, such as a human, must fuse the track and classification information to ID the correct target at a given time and can aid tracking algorithms by determining a set of tracks to follow and aid classification algorithms by constraining the set of plausible targets.

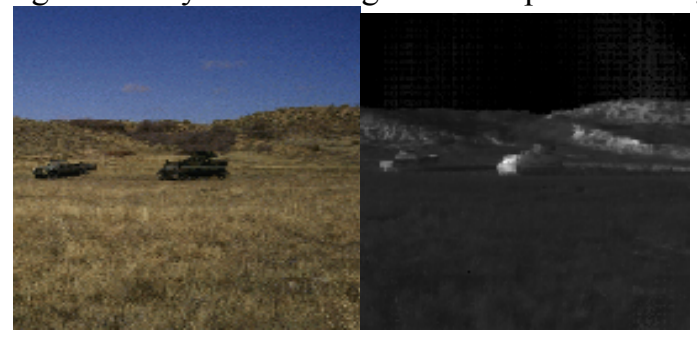

Figure 1. (a) Electro/Optical (EO) image and (B) Infrared (IR) image of targets [12]. 
Multitarget tracking in the presence of clutter has been investigated through the use of data association algorithms [1]. Likewise, other multisensor fusion algorithms have focused on tracking targets from multiple look sequences [13]. One inherent limitation of current algorithms is that the number of targets needs to be known a priori. While tracking algorithms can speculate on the number of targets, the cognition of the number of targets can be afforded to the user. The human is presented both the tracking information and the accrued evidence for each target type as a confidence measure. Once the human has an ID belief in the number of targets, the tracking algorithm can be updated. The human must then cognitively determine from the set of targets how many, what type, and which target goes with which track [14]. Additionally, the human has the ability to eliminate those targets that are not plausible which reduces the number of tracks and the set of pose templates from which the classification algorithm must search.

This paper presents a summary of DS methods in the last two decades. By introducing the operator or image analyst for cognitive fusion, it may offer a means to control some aspects of the computational burdens experienced by analytical data association techniques while improving track quality for multitarget tracking and ID in the presence of clutter. Section 2 overviews many applications of DS tracking. Section 3 describes DST methods while Section 4 compares the DST methods to Bayesian formulations. Section 5 presents a contemporary approach using the proportional conflict redistribution rule (PCR5) which is compared to the other methods in Section 6. Section 7 draws some conclusions.

\section{TRACKING METHODS USING DEMPSTER-SHAFER THEORY}

One of the earliest known works in applying Dempster-Shafer (DS) methods to target tracking was by Jean Dezert for navigation [15], where the sensor is moving and the targets are stationary. The emergence of the benefits of DS methods were applied by Robin Murphy for robotic scene analysis [16]. Building on Murphy's work, the DS methods were then applied to other robotic applications [17, 18, 19], albeit real-time control was still superior with Bayesian methods. At the same time, Johan Schubert applied DS methods for determining the number of tracks through the support and plausibility functions for the linking of submarine targets between tracks [20].

A few tracking and identification algorithms have been proposed for air-target tracking [21] and ground target tracking $[22,23]$ that extend Bayes' rule for identification where the most probable target is selected when there is incomplete knowledge. For instance, there are times when unknown targets might be of interest that are not known at algorithm initiation. At other times, there are unknown number of targets to track or targets not trained for classification. One way to study the problem is the interaction of the human and the machine working synergistically - since the sensors are extensions of the human's processing. The set theory approach to HRR target classification was proposed by Mitchell and Westerkamp and termed a Statistical feature based classifier (STaF) for air-target tracking [24]. In addition, Blasch [25, $26,27]$ presented a feature-based set-theory approach for ground target tracking. In both cases, classification and tracking, a set of features and a set of targets was investigated by extending the STaF algorithm as a belief filter for radar profiles analysis from which a plausible set of tracks and targets are made available to the user at each time instant [28].

Given the ability to track individual targets using advances in DS theory for target identify and classification, methods were then developed for tracking a group of targets. In this case, the like targets could be grouped together based on common characteristics [29, 30, 31]. Additionally, Li [32] investigated methods for convex optimization for enhanced ID processing. Using a combination of belief filtering and data association improved analysis of maneuvering targets [33, 34]. Other group tracking methods were postulated for cluster-to-track fusion [35]. Finally, the benefits of DS methods provided complementary information to tracking through Kalman weighting [36], mutual aiding [37] and pose estimation [38].

Beginning in 2005, efforts were made to extend traditional DS tracking methods [39] to that of advanced techniques using the proportional conflict redistribution rule (PCR5) [40]. Other tracking methods included multisensor [41], activity analysis [42], and out-of-sequence methods [43]. Also, at that time, methods of combining DS with nonlinear tracking methods such as the particle filter [44] and the unscented Kalman filter [45] were developed. Finally, a fusion rule based on DS methods was used to solve the association problem in target tracking [46].

With the demonstrated performance of many applications of DS techniques, research continued in the exploration of DS rules for classification to improve track accuracy [47] and maneuvering targets [48]. Multisensor techniques were applied for heterogeneous sensor measurements [49], such as DS tracking with unattended ground sensor measurements [50]. Currently, efforts are sought for tracking performance evaluation with DS techniques for tracking and identification improvement [51, 52, 53]. Further assessment includes combinations with non-linear tracking methods [54] and 
association of track segments [55]. More recent results have applied the developments from DS tracking from radar towards that of image processing [56, 57]. Throughout the many demonstrations of the successes of DS tracking, we now discuss DS methods, compare DST with Bayesian methods, and conclude with belief functions (BF) such as the contemporary PCR5 method for target tracking.

\section{BASICS OF DEMPSTER-SHAFER (DST) THEORY}

The Dempster-Shafer (DS) theory of evidence was devised as a means of dealing with imprecise evidence [58, 59] and has been applied to target classification [60]. Evidence concerning an unknown target is represented as a nonnegative set function $m: P(U) \rightarrow[0,1]$, where $P(U)$ denotes the set of subsets of the finite universe $U$ such that $\mathrm{m}(\varnothing)=0$ and $\Sigma_{S \subseteq U} \mathrm{~m}(S)$ $=1$. The set function $m$ is called a mass assignment and models a range of possible beliefs about propositional hypothesis of the general form $P_{\mathrm{S}} \triangleq$ "object is in $S_{1}$ " where $\mathrm{m}(S)$ is the weight of belief in the hypothesis $P_{\mathrm{S}}$. The quantity $\mathrm{m}(S)$ usually interprets as the degree of belief that accrues in $S$, but to no proper subset of $S$. The weight of belief $\mathrm{m}(U)$ attached to the entire universe is called the weight of uncertainty and models our belief in the possibility that the evidence $m$ in question is completely erroneous. The quantities

$$
\begin{aligned}
& B e l_{\mathrm{m}}(S) \triangleq \sum_{O \subseteq S} \mathrm{~m}(O) \\
& P l_{\mathrm{m}}(S) \triangleq \sum_{O \cap S \neq \varnothing} \mathrm{m}(O)
\end{aligned}
$$

are called the belief and plausibility of the evidence, respectively and $\mathrm{m}(O)$ is the mass assignment for object $O$. The relationships $\operatorname{Bel}_{\mathrm{m}}(S) \leq P l_{\mathrm{m}}(S)$ and $\operatorname{Bel}_{\mathrm{m}}(S)=1-P l_{\mathrm{m}}\left(S^{\mathrm{c}}\right)$ are true identically and the interval $\left[\operatorname{Bel}_{\mathrm{m}}(S), P l_{\mathrm{m}}(S)\right]$ is called the interval of uncertainty, $\left(I O U_{\mathrm{m}}\right)$. Knowing that $B_{e} l_{\mathrm{m}}(S) \rightarrow[0,1]$ and $P l_{\mathrm{m}}(S) \rightarrow[0,1]$, three relationships exist. The first is a direct use of $\mathrm{Bel}_{\mathrm{m}}(S)$ to accept and $P l_{\mathrm{m}}(S)$ to reject measurements. Another method is to use the interval of certainty (IOC) defined as $[1,1]-\left[\operatorname{Bel}_{\mathrm{m}}(S), P l_{\mathrm{m}}(S)\right]$ using interval subtraction. For example, $[1,1]-[0.8,0.9]=[1-0.9,1-0.8]=[0.1,0.2]$. Using the lower bound $B e l_{\mathrm{m}}(S)$ and upper bounds $P l_{\mathrm{m}}(S)$ of the interval, we can assign a confidence measure $C_{\mathrm{m}}=1+B e l_{\mathrm{m}}$ $(S)-P l_{\mathrm{m}}(S)=1+0.8-0.9=0.9$. Finally, the $I O U_{\mathrm{m}}=\left[\operatorname{Bel}_{\mathrm{m}}(S), P l_{\mathrm{m}}(S)\right]$ can be mapped to a Gaussian distribution for beliefbased track tracking, using the $I O U_{\mathrm{m}}$ center as the mean, $\mu$, rescaling the bounds to a Normal distribution and tacking the estimates (mean and variance). For example, $\mu_{\mathrm{m}}=(0.8+0.9) / 2=0.85$. As another example, assume low belief with many measurements plausible, then $\operatorname{Bel}_{\mathrm{m}}(S)=0.3$ and $P l_{\mathrm{m}}(S)=0.9$, where $C_{\mathrm{m}}=0.6$ is lower and the mean is $\mu_{\mathrm{m}}=0.6$.

The mass assignment can be recovered from the belief function via the Möbius transform: [61]

$$
\mathrm{m}(S) \triangleq \sum_{O \subseteq S}(-1)^{|S-O|} B l_{\mathrm{m}}(O)
$$

The set intersection quantity

$$
(\mathrm{m} \oplus \mathrm{n})(S) \triangleq \frac{1}{1-K} \sum_{X \cap Y=\mathrm{S}} \mathrm{m}(X(O)) \mathrm{n}(Y(O))
$$

is called Dempster's rule of combination, where $K \triangleq \sum_{X(O) \cap Y(O)=\varnothing} \mathrm{m}(X(O)) \mathrm{n}(Y(O))$ is called the conflict between the evidence $m$ and evidence $n$.

In the finite-universe case, the Dempster-Shafer theory (DST) coincides with the theory of independent, non empty subsets of $U$ (see [62, 63]; or for a dissenting view, see [64]). Given a mass assignment $m$, it is always possible to find a random subset $\Sigma$ of $U$ such that $\mathrm{m}(S)=p(\Sigma=S)$. In this case, $\operatorname{Bel}_{\mathrm{m}}(S)=p(\Sigma \subseteq S)=\beta_{\Sigma}(S)$ and $P l_{\mathrm{m}}(S)=p(\Sigma \cap S \neq 0)=\rho_{\Sigma}(S)$ where $\beta_{\Sigma}$ and $\rho_{\Sigma}$ are the belief and plausibility measures of $\Sigma$, respectively. Likewise, we can construct independent random subsets, $\Sigma, \Lambda$ of $U$ such that $\mathrm{m}(S)=p(\Sigma=\mathrm{S})$ and $\mathrm{n}(\mathrm{S})=p(\Lambda=\mathrm{S})$ for all $S \subseteq U$. Then, it is easy to show that 
$(\mathrm{m} \oplus \mathrm{n})(S)=p(\Sigma \cap \Lambda \mid \Sigma \cap \Lambda \neq 0)$

for all $S \subseteq U$. Thus, an intersection of overlapping sets can be fused to generate a global set confidence, where confidence is defined on the range $[1,1]-\left[\mathrm{Bel}_{\mathrm{m}}, P l_{\mathrm{m}}\right]$ and uncertainty is $\left[\mathrm{Bel}_{\mathrm{m}}, P l_{\mathrm{m}}\right]$. As comparative to the optimal approach, the next section provides a comparison of the set theory approach to that of traditional Bayesian analysis.

\section{DEMPSTER-SHAFER VERSUS BAYESIAN THEORY}

Recently, Dezert [65] has shown that Dempster's rule is consistent with probability calculus and Bayesian reasoning if and only if the prior $P(X)$ is uniform. However, when the $P(X)$ is not uniform, then Dempster's rule gives a different result. Both Yen [66] and Mahler [67, 68] developed methods to account for non-uniform priors. Others have also tried to compare Bayes and DST methods $[69,70,71,72,73,74,75]$. Assuming that we have multiple measurements $Z=\left\{Z_{1}, Z_{2}, \ldots\right\}$ for object $O$ being tracked, Bayesian and DS methods are developed next.

Assuming conditional independence, one has the Bayes method:

$$
\mathrm{P}\left(X \mid Z_{1} \cap Z_{2}\right)=\frac{\mathrm{P}\left(X \mid Z_{1}\right) \mathrm{P}\left(X \mid Z_{2}\right) / \mathrm{P}(X)}{\sum_{\mathrm{i}=1}^{\mathrm{N}} \mathrm{P}\left(X_{\mathrm{i}} \mid Z_{1}\right) \mathrm{P}\left(X_{\mathrm{i}} \mid Z_{2}\right) / \mathrm{P}\left(X_{\mathrm{i}}\right)}
$$

With no information from $Z_{1}$ or $Z_{2}$, then $\mathrm{P}\left(X \mid Z_{1}, Z_{2}\right)=\mathrm{P}(X)$. Without $Z_{2}$, then $\mathrm{P}\left(X \mid Z_{1}, Z_{2}\right)=\mathrm{P}\left(X \mid Z_{1}\right)$ and without $Z_{1}$, then $\mathrm{P}\left(X \mid Z_{1}, Z_{2}\right)=\mathrm{P}\left(X \mid Z_{2}\right)$. Using Dezert's formulation, then the denominator can be expressed as a normalization coefficient:

$$
m_{12}(\varnothing)=1-\sum_{\mathrm{X}_{\mathrm{i}} ; \mathrm{X}_{\mathrm{j}} \mid \mathrm{X}_{\mathrm{i}} \cap \mathrm{X}_{\mathrm{j}}} \mathrm{P}\left(X_{\mathrm{i}} \mid Z_{1}\right) \mathrm{P}\left(X_{\mathrm{i}} \mid Z_{2}\right)
$$

Using this relation, then the total probability mass of the conflicting information is

$$
\mathrm{P}\left(X \mid Z_{1} \cap Z_{2}\right)=\frac{1}{1-m_{12}(\varnothing)} \bullet \mathrm{P}\left(X \mid Z_{1}\right) \mathrm{P}\left(X \mid Z_{2}\right)
$$

which corresponds to Dempster's rule of combination using Bayesian belief masses with uniform priors. When the prior's are not uniform, then Dempster's rule is not consistent with Bayes' Rule. For example, let $m_{0}(X)=P(X), m_{1}(X)=P\left(X \mid Z_{1}\right)$, and $m_{2}(X)=P\left(X \mid Z_{2}\right)$, then

$$
m(X)=\frac{m_{0}(X) m_{1}(X) m_{2}(X)}{1-m_{012}(\varnothing)}=\frac{\mathrm{P}(X) \mathrm{P}\left(X \mid Z_{1}\right) \mathrm{P}\left(X \mid Z_{2}\right)}{\sum_{\mathrm{i}=1}^{\mathrm{N}} \mathrm{P}\left(X_{\mathrm{i}}\right) \mathrm{P}\left(X_{\mathrm{i}} \mid Z_{1}\right) \mathrm{P}\left(X_{\mathrm{i}} \mid Z_{2}\right)}
$$

Thus, methods are needed to deal with non-uniform priors and appropriately redistribute the conflicting masses.

\section{DEZERT-SMARANDACHE THEORY (DSmT)}

Recent advances in DS methods include Dezert-Smarandache Theroy (DSmT). DSmT is an extension to the DempsterShafer method of evidential reasoning which has been detailed in numerous papers and texts: Advances and applications of DSmT for information fusion (Collected works), Vols. 1-3 [76]. In 2002, Dezert [77] introduced the methods for the reasoning and in 2003, presented the hyper power-set notation for DSmT [78]. Recent applications include the DSmT Proportional Conflict Redistribution rule 5 (PCR5) applied to target tracking. Other applications of DSmT can be found in the list of references at (http://www.onera.fr/staff/jean-dezert/) .

The key contributions of DSmT are the redistributions of masses such that no refinement of the frame $\Theta$ is possible unless a series of constraints are known. For example, Shafer's model [79] is the most constrained DSm hybrid model in DSmT. 
Since Shafer's model, authors have continued to refine the method to more precisely address the combination of conflicting beliefs $[80,81,82]$ and generalization of the combination rules [83, 84]. An adaptive combination rule [85] and rules for quantitative and qualitative combinations [86] have been proposed. Recent examples for sensor applications include electronic support measures, [87, 88], physiological monitoring sensors [89], and seismic-acoustic sensing [90].

Here we use the Proportional Conflict Redistribution rule no. 5 (PCR5) and no. 6 (PCR6) and the Dezert-Smarandache Probability (DSmP) selections which are discussed below. We replace Smets' rule [80] by the more effective PCR5 or eventually the more simple PCR6 and replace the pignistic transformation by the more effective DSmP transformation to estimate target classification probabilities. All details, justifications with examples on PCR5 and PCR6 fusion rules and DSmP transformation can be found freely from the web in the DSmT compiled texts [76], Vols. $2 \& 3$.

\subsection{PCR5 and PCR6 fusion rules}

In the DSmT framework, the PCR5 is used generally to combine the basic belief assignment (bba)'s. PCR5 transfers the conflicting mass only to the elements involved in the conflict and proportionally to their individual masses, so that the specificity of the information is entirely preserved in this fusion process. Let $m_{1}($.$) and m_{2}($.$) be two independent bba's, then$ the PCR5 rule is defined as follows (see [76], Vol. 2 for full justification and examples): $m_{\mathrm{PCR} 5}(\varnothing)=0$ and $\forall X \in 2^{\Theta} \backslash\{\varnothing\}$, where $\varnothing$ is the null set and $2^{\Theta}$ is the power set:

$$
m_{\mathrm{PCR} 5}(X)=\sum_{\substack{\mathrm{X}_{1} ; \mathrm{X}_{2} \in 2^{\Theta} \\ \mathrm{X}_{1} \cap \mathrm{X}_{2}=\mathrm{X}}} m_{1}\left(X_{1}\right)+m_{2}\left(X_{2}\right)+\sum_{\substack{\mathrm{X}_{2} \in 2^{\Theta} \\ \mathrm{X}_{2} \cap \mathrm{X}=\varnothing}}\left[\frac{m_{1}\left(X_{1}\right)^{2} m_{2}\left(X_{2}\right)}{m_{1}\left(X_{1}\right)+m_{2}\left(X_{2}\right)}+\frac{m_{1}\left(X_{1}\right) m_{2}\left(X_{2}\right)^{2}}{m_{1}\left(X_{1}\right)+m_{2}\left(X_{2}\right)}\right]
$$

where $\bigcap$ is the interesting and all denominators in the equation above are different from zero. If a denominator is zero, that fraction is discarded. Additional properties and extensions of PCR5 for combining qualitative bba's can be found in [76], Vol. 2 \& 3. All propositions/sets are in a canonical form. A variant of PCR5, called PCR6 has been proposed by Martin and Osswald in [91], Vol. 2, for combining more than 2 sources. PCR6 coincides with PCR5 when one combines two sources. The difference between PCR5 and PCR6 lies in the way the proportional conflict redistribution is done as soon as three or more sources are involved in the fusion. For example, let's consider three sources with bba's $m_{1}(),. m_{2}($.$) , and m_{3}(),. A \cap B=$ $\varnothing$ for the model of the frame $\Theta$, and $m_{1}(A)=0.6, m_{2}(B)=0.3$, and $m_{3}(B)=0.1$. With $\boldsymbol{P C R} 5$ the partial conflicting mass $m_{1}(A) m_{2}(B) m_{3}(B)=(0.6)(0.3)(0.1)=0.018$ is redistributed back to $A$ and $B$ only with respect to the following proportions respectively: $x_{\mathrm{A}}{ }^{\mathrm{PC} 5}=0.01714$ and $x_{\mathrm{B}}{ }^{\mathrm{PCR} 5}=0.00086$ because the proportionalization is:

$$
\frac{x_{\mathrm{A}}^{\mathrm{PCR} 5}}{m_{1}(A)}=\frac{x_{\mathrm{B}}{ }^{\mathrm{PCR} 5}}{m_{2}(B) m_{3}(B)}=\frac{m_{1}(A) m_{2}(B) m_{3}(B)}{m_{1}(A)+m_{2}(B) m_{3}(B)}
$$

that is $\quad \frac{x_{\mathrm{A}}{ }^{\mathrm{PCR} 5}}{0.6}=\frac{x_{\mathrm{B}}^{\mathrm{PCR} 5}}{(0.3)(0.1)}=\frac{0.018}{0.6+0.03} \approx 0.02857$

thus $\quad x_{\mathrm{A}}{ }^{\mathrm{PCR} 5}=0.60(0.02857) \approx 0.01714$

$$
x_{\mathrm{B}}^{\mathrm{PCR} 5}=0.03(0.02857) \approx 0.00086
$$

With the PCR6 fusion rule, the partial conflicting mass $m_{1}(A) m_{2}(B) m_{3}(B)=(0.6)(0.3)(0.1)=0.018$ is redistributed back to $A$ and $B$ only with respect to the following proportions respectively: $x_{\mathrm{A}}{ }^{\mathrm{PCR} 6}=0.0108$ and $x_{\mathrm{B}}{ }^{\mathrm{PCR} 6}=0.0072$ because the PCR6 proportionalization is done as follows:

$$
\frac{x_{\mathrm{A}}^{\mathrm{PCR} 6}}{m_{1}(A)}=\frac{x_{\mathrm{B}: 2}{ }^{\mathrm{PCR} 6}}{m_{2}(B)}=\frac{x_{\mathrm{B}: 3}{ }^{\mathrm{PCR} 6}}{m_{3}(B)}=\frac{m_{1}(A) m_{2}(B) m_{3}(B)}{m_{1}(A)+m_{2}(B)+m_{3}(B)}
$$

that is

$$
\frac{x_{\mathrm{A}}^{\mathrm{PCR} 6}}{0.6}=\frac{x_{\mathrm{B}: 2}^{\mathrm{PCR} 6}}{0.3}=\frac{x_{\mathrm{B}: 3}{ }^{\mathrm{PCR} 6}}{0.1}=\frac{0.018}{0.6+0.3+0.1} \approx 0.018
$$

thus 


$$
\begin{aligned}
& x_{\mathrm{A}}{ }^{\text {PCR6 }}=(0.6)(0.018)=0.0108 \\
& x_{\mathrm{B}, 2}{ }_{\text {PCR } 6}=(0.3)(0.018)=0.0054 \\
& x_{\mathrm{B}, 3}{ }^{\text {PCR }}=(0.1)(0.018)=0.0018
\end{aligned}
$$

and therefore with PCR6, one gets finally the following redistributions to $A$ and $B$ :

$$
\begin{aligned}
& x_{\mathrm{A}}{ }^{\text {PCR6 }}=(0.6)(0.018)=0.0108 \\
& x_{\mathrm{B}}{ }^{\text {PCR6 }}=x_{\mathrm{B}, 2}{ }^{\text {PCR6 }}+x_{\mathrm{B}, 3}{ }^{\text {PCR6 }}=0.0054+0.0018=0.0072
\end{aligned}
$$

From the implementation point of view, PCR6 is simpler to implement than PCR5. For convenience, Matlab codes of PCR5 and PCR6 fusion rules can be found in [76]. It is worth noting that there is a strong relationship between PCR6 rule and the averaging fusion rule which is commonly used to estimate the probabilities in the classical frequentist interpretation of probabilities. Such a probability estimate cannot be obtained using DS rule, nor the PCR5 rule and that is why we recommend to use PCR6 when combining more than two basic belief masses altogether [92].

\subsection{The DSmP Transformation}

The DSmP probabilistic transformation is an alternative to the classical pignistic transformation which allows us to increase the probabilistic information content (PIC), i.e. to minimize the Shannon entropy, of the approximated subjective probability measure drawn from any bba. Justification and comparisons of $\mathrm{DSmP}($.$) with respect to \operatorname{BetP}($.$) and to other transformations$ can be found in details in [93, 76 Vol. 3, Chap. 3].

BetP: The pignistic transformation probability, denoted BetP, offers a compromise between maximum of credibility $\mathrm{Bel}$ and maximum of plausibility $P l$ for decision support. The $\operatorname{Bet} P$ transformation is defined by $\operatorname{BetP}(\varnothing)=0$ and $\forall X \in \mathrm{G}^{\Theta} \backslash\{\varnothing\}$ by

$$
\operatorname{Bet} P(X)=\sum_{\mathrm{Y} \in \mathrm{G}}{ }^{\Theta} \frac{C_{\mathrm{M}}(X \cap Y)}{C_{\mathrm{M}}(Y)} m(Y)
$$

where $G^{\Theta}$ corresponds to the hyper-power set including all the integrity constraints of the model (if any). $G^{\Theta}=2^{\Theta}$ if one adopts Shafer's model for $\Theta$ and $\mathrm{G}^{\Theta}=\mathrm{D}^{\Theta}$ (Dedekind's lattice) if one adopts the free DSm model for $\Theta[76]$. $C_{\mathrm{M}}(Y)$ denotes the DSm cardinal of the set $Y$, which is the number of parts of $Y$ in the Venn diagram of the model $M$ of the frame $\Theta$ under consideration [76, Book 1, Chap. 7]. The BetP reduces to the Transferable Belief Model (TBM) when $G^{\Theta}$ reduces to classical power set $2^{\Theta}$ when one adopts Shafer's model.

DSmP transformation is defined by $\operatorname{DSmP}_{\varepsilon}(\varnothing)=0$ and $\forall X \in \mathrm{G}^{\Theta} \backslash\{\varnothing\}$ by:

$$
D S m P_{\mathcal{E}}(X)=\sum_{\mathrm{Y} \in \mathrm{G}} \Theta \frac{\sum_{\substack{\mathrm{Z} \subseteq \mathrm{X} \cap \mathrm{Y} \\ \mathrm{C}(\mathrm{Z})=1}} m(Z)+\varepsilon \bullet C(X \cap Y)}{\sum_{\substack{\mathrm{Z} \subseteq \mathrm{Y} \\ \mathrm{C}(\mathrm{Z})=1}} m(Z)+\varepsilon \bullet C(Y)} m(Y)
$$

where $\mathrm{C}(X \cap Y)$ and $\mathrm{C}(Y)$ denote the cardinals of the sets $X \cap Y$ and $Y$ respectively; $\varepsilon \geq 0$ is a small number which allows to reach a highest PIC value of the approximation of $m($.$) into a subjective probability measure, and Z$ is the new evidence. Usually $\varepsilon=0$, but in some particular degenerate cases, when the $\mathrm{DSmP}_{\mathcal{\varepsilon}=0}$ (.) values cannot be derived, the $\mathrm{DSmP}_{\mathcal{\varepsilon}>0}$ values can however always be derived by choosing $\varepsilon$ as a very small positive number, say $\varepsilon=1 / 1000$ for example in order to be as close as we want to the highest value of the PIC. The smaller $\varepsilon$, the better/bigger PIC value one gets. When $\varepsilon=1$ and when the masses of all elements $Z$ having $\mathrm{C}(Z)=1$ are zero, $\operatorname{DSmP}_{\mathcal{E}=1}()=.\operatorname{BetP}($.$) .$ 


\section{COMPARATIVE RESULTS OF DS, BAYES, AND PCR5-BASED TRACKING}

Here we simulate two scenarios of the three rules: Bayesian, Dempster-Shafer, and PCR5 rules of combination. For each scenario, we assume that the target information is collected from a sensor that is precise in the position measurements, but the uncertainty in either the sensor position accuracy or the classification information results in a confusion matrix (CM) formulation. With a two object representation being tracked (e..g, the standard fighter/cargo example), we have $C M=\left[O_{1}\right.$ $\left.\mathrm{O}_{2} ; \mathrm{O}_{2} \mathrm{O}_{1}\right]$. In the first scenario, the target classification is $C M=\left[\begin{array}{lll}0.750 .25 ; 0.25 & 0.75\end{array}\right]$, and the belief mass results are shown in Figure 2.
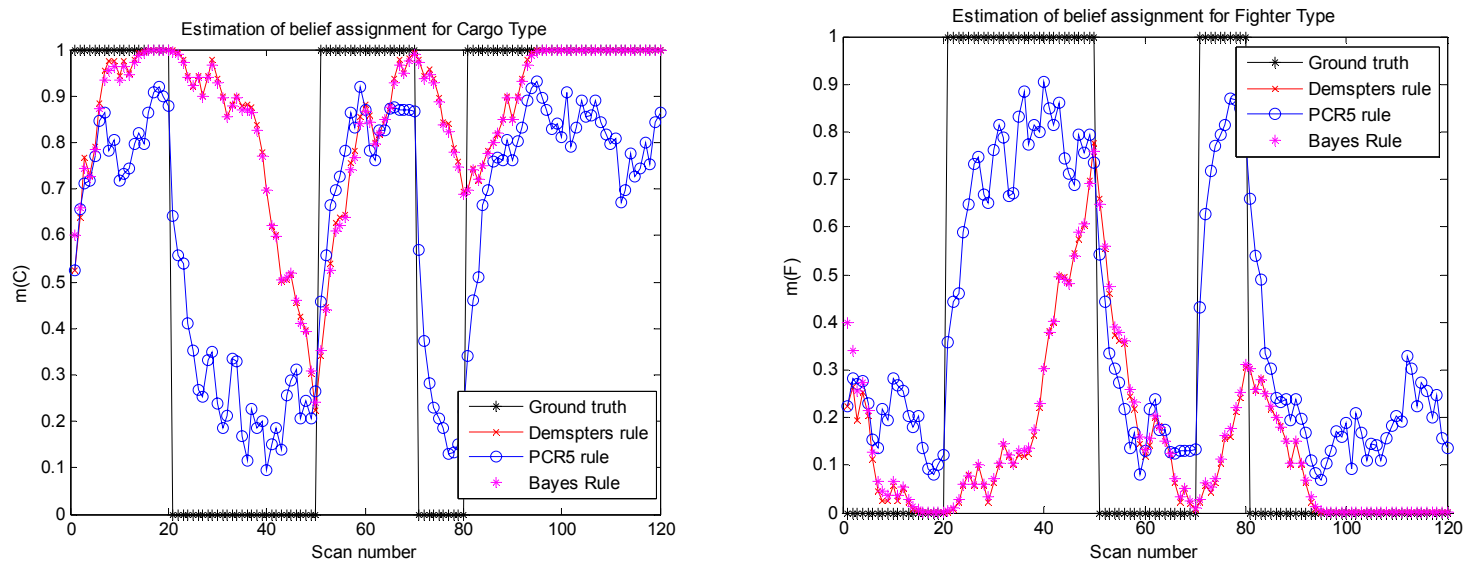

Figure 2. (a) Object 1 and (b) Object 2 tracking and identification results. $C M=\left[\begin{array}{lllll}0.75 & 0.25 ; & 0.25 & 0.75\end{array}\right]$

Figure 2 demonstrates that while there is uncertainty in the object tracking and classification, both the DS and Bayesian methods are close. The PCR5 results in better accuracy. Both DS and Bayesian methods have difficulty when the measurements change and suffer from a prior evidence biasing. In the next scenario, we decrease the sensor classification/ID accuracy; which results in more conflict in the analysis. For the Scenario 2 sensor model, we use $C M=$ $[0.650 .35 ; 0.350 .65]$.
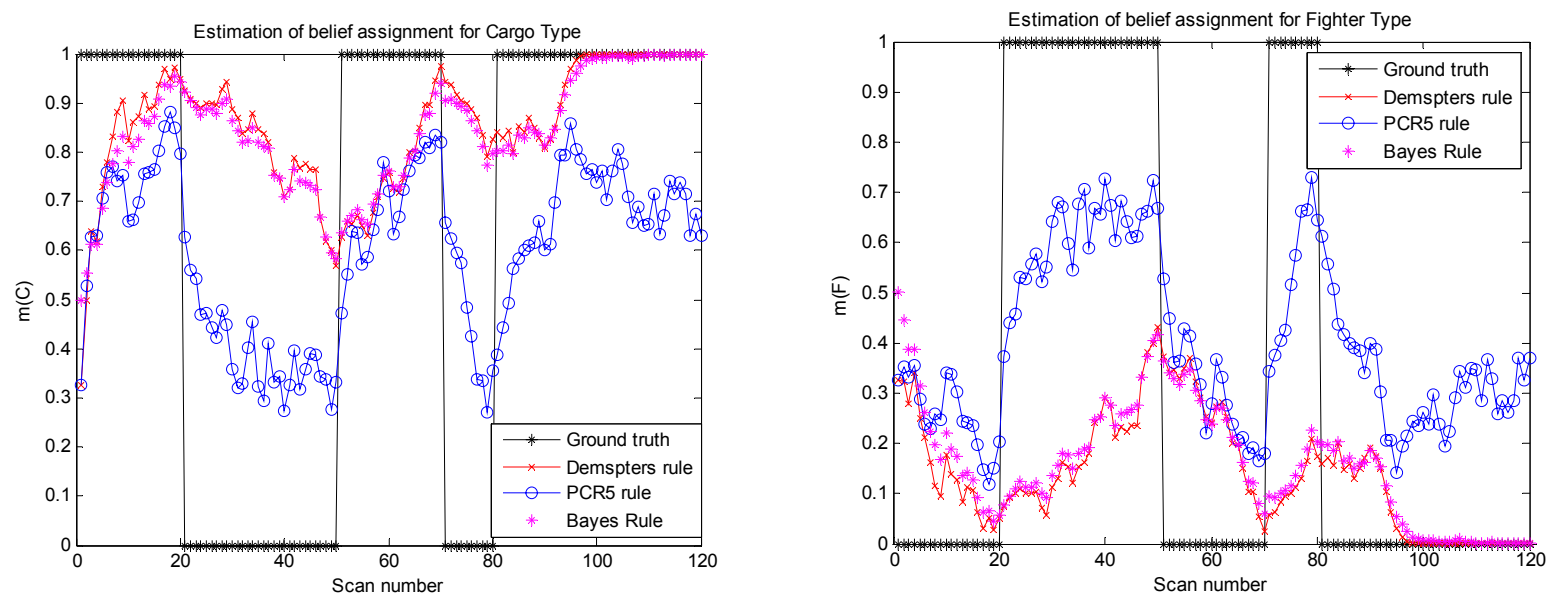

Figure 3. (a) Object 1 and (b) Object 2 tracking and identification results. $C M=\left[\begin{array}{lllll}0.65 & 0.35 ; & 0.35 & 0.65\end{array}\right]$.

Figure 3 illustrates differences between the three methods. DS tracking methods are able to improve over standard Bayesian methods when there is conflict in the measurements (Fig 3a scan 10 to 20). However, as shown in Figure 3, the PCR5 demonstrates an ability to track and ID the target when the measurement information is conflicting and changing (Fig 3a scan 25 to 50). The simple example illustrates the power of the PCR5 rule over standard DS and Bayesian methods to deal with conflicting, imprecise, and variations in target measurements for target tracking. 


\section{CONCLUSIONS}

Conventional tracking techniques have difficulty in identifying targets when the number of targets is not known a priori., the targets are maneuvering, and there is conflict in the measurements. Throughout the last two decades, numerous researchers have explored Dempster-Shafer (DS) evidential (i.e., belief function) reasoning to solve the requirements of simultaneous tracking and identification. This paper has provided a literature review of most of the available publications that utilize the DS method in target, group/cluster, and multisensor tracking. Through a review of Bayesian, DS, and PCR5 formulations; we presented a simulated comparative example to demonstrate the current state-of-the-art methods such as DSmT research [94]. The PCR5 method can be extended to nonlinear tracking and ID algorithms, coordinated with users for assisted tracking, and can enhance conventional covariance and information filter formulations [95]. The presented PCR5 technique demonstrates promise for multitarget tracking problems and warrants further exploration with real-world data where environmental effects, occlusions, lost sensor data, and unknown targets [96] are standard.

\section{REFERENCES}

[1] Blasch, E. P., Bossé, E., and Lambert, D. A., [High-Level Information Fusion Management and Systems Design], Artech House, Norwood, MA, (2012).

[2] Kahler, B., and Blasch, E., "Decision-Level Fusion Performance Improvement from Enhanced HRR Radar Clutter Suppression,” J. of. Advances in Information Fusion, Vol. 6, No. 2, Dec. (2011).

[3] Waltz, E., and Llinas, J., [Multisensor Data Fusion], Artech House, (1990).

[4] Bar-Shalom, Y., and Li, X. R., [Multitarget-Multisensor Tracking: Principles and Techniques], YBS, New York, (1995).

[5] Duda, R. O., Hart, P. E., and Stork, D. G., [Pattern Classification (2nd Edition)], Wiley, (2000).

[6] Blasch, E. P., and Watamaniuk, S. N., "Cognitive-based fusion using information sets for moving target recognition," Proc. SPIE, Vol. 4052, (2000).

[7] Blasch, E. P., “Assembling a distributed fused Information-based Human-Computer Cognitive Decision Making Tool," IEEE Aerospace and Electronic Systems Magazine, Vol. 15, No. 5, pp. 11-17, May (2000).

[8] Blasch, E., "Situation, Impact, and User Refinement," Proc. of SPIE, Vol. 5096, (2003).

[9] Blasch, E., "Level 5 (User Refinement) issues supporting Information Fusion Management" Int. Conf. on Info Fusion, (2006).

[10] Blasch, E., Kadar, I., Salerno, J., Kokar, M. M., Das, S., Powell, G. M., Corkill, D. D., and Ruspini, E. H., "Issues and Challenges in Situation Assessment (Level 2 Fusion)," J. of Advances in Information Fusion, Vol. 1, No. 2, pp. 122 - 139, Dec. (2006).

[11] Blasch, E., "Sensor, User, Mission (SUM) Resource Management and their interaction with Level 2/3 fusion" Int. Conf. on Info Fusion, (2006).

[12] Blasch, E., and Kahler, B., "Multi-resolution EO/IR Tracking and Identification” Int. Conf. on Info Fusion, (2005).

[13] Blasch, E., and Gainey, J., "Feature Based Biological Sensor Fusion," Intl. Conference on Info. Fusion, (1998).

[14] Blasch, E. P. and Plano, S. B., "JDL Level 5 Fusion model 'user refinement' issues and applications in group Tracking," Proc. SPIE, Vol. 4729, (2002).

[15] Dezert, J., "Autonomous navigation with uncertain reference points using the PDAF," in Multitarget-Multisensor Tracking, Vol 2, pp 271-324, Y. Bar-Shalom (Ed), Artech House, (1991).

[16] Murphy, R. R., "Dempster-Shafer Theory for Sensor Fusion in Autonomous Mobile Robots," IEEE Tr. On Robotics and Automation, Vol. 14, No. 2, April (1998).

[17] Blasch, E., Kondor, S., Gordon, M., and Hsu, R., "Georgia Tech Aerial Robotics team competition entry," J. Aerial Unmanned Vehicle Systems, pp. 20 - 25, May (1994).

[18] Blasch, E., "Flexible Vision-Based Navigation System for Unmanned Aerial Vehicles," Proc. SPIE, Vol. 2352, (1995).

[19] Balch, T., Boone, G., Collins, T., Forbes, H., MacKenzie, D., and Santamaria, J.C., "Io, Ganymede, and Callisto a multiagent robot trash-collecting team," AI Magazine, 16 (2): 39-53, (1995).

[20] Schubert, J, [Cluster-based Specification Techniques in Dempster-Shafer Theory for an Evidential Intelligence Analysis of Multiple Target Tracks], Ph.D. thesis, TRITA-NA-9410, Dept. of Numerical Analysis and Computing Science, Royal Institute of Technology, Stockholm, (1994).

[21] Leung, H., Li. Y., Bossé, E., Blanchette, M., Chan, K.C.C, "Improved multiple target tracking using Dempster-Shafer Identification," Proc. SPIE, Vol. 3068, (1997).

[22] Blasch, E., and Hong, L., "Simultaneous Tracking and Identification," IEEE Conference on Decision Control, pg. 249-256, (1998).

[23] Ding, Z., and Hong, L., "Decoupling probabilistic data association algorithm for multiplatform multisensor tracking," Optical Engineering, Vol. 37, No. 2, Feb. (1998).

[24] Micthell, R. A., and Westerkamp, J. J., "Robust Statistical Feature-based aircraft identification," IEEE. TR. on Aerospace and Electronics Systems, Vol. 35, No. 3, 1077-1094, (1999).

[25] Blasch, E., [Derivation of a Belief Filter for Simultaneous High Range Resolution Radar Tracking and Identification], Ph.D. Thesis, Wright State University, (1999).

[26] Blasch, E, Hong, L., "Sensor Fusion Cognition using belief filtering for tracking and identification,” Proc. SPIE, Vol. 3719, (1999). 
[27] Blasch, E. P., Westerkamp, J.J., Layne, J.R., Hong, L., Garber, F. D., and Shaw, A., "Identifying moving HRR signatures with an ATR Belief Filter," Proc. SPIE, Vol. 4053, (2000).

[28] Blasch, E., Hong, L., "Data Association through Fusion of Target track and Identification Sets," Int. Conf. on Info Fusion, (2000).

[29] Blasch, E., Connare, T., "Improving track accuracy through Group Information Feedback," Int. Conf. on Info Fusion, (2001).

[30] Blasch, E., Connare, T., "Improving Track maintenance Through Group Tracking," Proc of the Workshop on Estimation, Tracking, and Fusion; A Tribute to Yaakov Bar Shalom, Monterey, CA, 360 -371, May (2001).

[31] Connare, T., Blasch, E., Schmitz, J., Salvatore, F., Scarpino, F., "Group IMM tracking utilizing Track and Identification Fusion," Proc. of the Workshop on Estimation, Tracking, and Fusion; A Tribute to Yaakov Bar Shalom, 205 -220, May (2001).

[32] Li, J., Luo, Z-Q.,Wong, K.M., Bossé, E., "Convex optimization approach to identify fusion for multisensor target tracking," IEEE Transactions on Systems, Man and Cybernetics, Part A: Systems and Humans, Volume: 31, pp. 172 - 178, (2001).

[33] Blasch, E., "Information-Theory-based feature-aided tracking and identification algorithm for tracking moving and stationary targets through high-turn maneuvers using fusion of SAR and HRR information”, Proc. of SPIE, Vol. 4727, April (2002).

[34] Blasch, E., Connare, T., "Feature-Aided JBPDAF group tracking and classification using an IFFN sensor," Proc. SPIE, Vol. 4728, (2002).

[35] Schubert, J., "Robust report level cluster-to-track fusion," Int'l Conf. on Information Fusion, (2002).

[36] Wu, H., Siegel, M. ; Ablay, S., "Sensor fusion using Dempster-Shafer theory II: static weighting and Kalman filter-like dynamic weighting," IEEE Proc. Instrumentation and Measurement Technology Conference, (2003).

[37] Yang, C., and Blasch, E., "Mutual Aided Target Tracking and Identification," Proc. of SPIE, Vol. 5099, (2003).

[38] Yang, C., and Blasch, E., "Pose Angular-Aiding for Maneuvering Target Tracking”, Int. Conf. on Info Fusion, (2005).

[39] Dezert, J., Tchamova, A. ; Semerdjiev, T., Konstantinova, P., "Performance evaluation of fusion rules for multitarget tracking in clutter based on generalized data association," Int'l Conf. on Information Fusion, (2005).

[40] Dezert, J., Tchamova, A., Smarandache, F., Konstantinova, P., “Target Type Tracking with PCR5 and Dempster's rules: A Comparative Analysis," Int'l Conf. on Information Fusion, (2006).

[41] Lancaster, J., Blackman, S, “Joint IMM/MHT Tracking and Identification for Multi-Sensor Ground Target Tracking," Int'l Conf. on Information Fusion, (2006).

[42] Snidaro, L., Piciarelli, C., Foresti, G.L., "Activity Analysis for Video Security Systems," IEEE International Conference on Image Processing, (2006).

[43] Maskell, S. R., Everitt, R. G., Wright, R., Briers, M., "Multi-target out-of-sequence data association: Tracking using graphical models," Information Fusion, Volume 7, Issue 4, Pages 434-447, December (2006).

[44] Faux, F., and Luthon, F., "Robust face tracking using color Dempster-Shafer fusion and particle filter," Int'l Conf. on Information Fusion, (2006).

[45] Khairnar, D.G., Nandakumar, S. , Merchant, S.N., Desai, U.B., "Nonlinear Target Identification and Tracking Using UKF," IEEE Conf on Granular Computing, (2007).

[46] Tchamova, A, Dezert, J., Smarandache, F., "A new class fusion rule for solving Blackman's Association Problem," International IEEE Conference Intelligent Systems, (2008).

[47] Kouemou, G., Neumann, C., Opitz, F., "Exploitation of track accuracy information in fusion technologies for radar target classification using Dempster-Shafer Rules," Int'l Conf. on Information Fusion, (2009).

[48] Dezert, J., Pannetier, B., “A PCR-BIMM filter for maneuvering target tracking," Int. Conf. on Info Fusion, (2010).

[49] Munz, M., Dietmayer, K., Mählisch, M., "Generalized fusion of heterogeneous sensor measurements for multi target tracking," Int'l Conf. on Information Fusion, (2010).

[50] Blasch, E., Maupin, P., Jousselme, A-L., "Sensor-Based Allocation for Path Planning and Area Coverage Using UGSs," Proc. IEEE Nat. Aerospace Electronics Conf (NAECON), (2010).

[51] Blasch, E., Valin, P., "Track Purity and Current Assignment Ratio for Target Tracking and Identification Evaluation,” Int. Conf. on Info Fusion, (2011).

[52] Pannetier, B., Dezert, J., "Extended and Multiple Target Tracking: Evaluation of an Hybridized Solution," Int'l Conf. on Info Fusion, (2011).

[53] Blasch, E., Straka, O., Yang, C., Qiu, D., Šimandl, M., Ajgl, J., "Distributed Tracking Fidelity-Metric Performance Analysis Using Confusion Matrices," Int. Conf. on Info Fusion, (2012).

[54] Liu, X., Leung, H., Valin, P., Bossé, E., "Multisensor joint tracking and identification using particle filter and Dempster-Shafer fusion," Int'l Conf. on Information Fusion, (2012).

[55] Pannetier, B., Dezert, J., "Track Segment Association with Classification Information," Workshop on Sensor Data Fusion: Trends, solution, Applications, 60-65, (2012).

[56] Dallil, A., Oussalah, M., Ouldali, A., "Sensor Fusion and Target Tracking Using Evidential Data Association,” IEEE Sensors Journal Volume: 13 , Issue: 1, pp. 285 - 293, (2013).

[57] Li, X., Dick, A., Shen, C., Zhang, Z., Vandenhengel, A., Wang, H., "Visual Tracking with Spatio-Temporal Dempster-Shafer Information Fusion," IEEE Tr. on Image Processing, (2013).

[58] Buede, D., "Shafer-Dempster and Bayesian reasoning: A response to 'Shafer-Dempster reasoning with applications to multisensor target identification," IEEE Transaction on Syst., Man and Cyber., vol.18, pp.1-10, (19880.

[59] Hall, D. L., [Mathematical Techniques in Multisensor Data Fusion], Artech House, (1992).

[60] Kruse, R., Schwencke, E., Heinsohn, J., [Uncertainty and Vagueness in Knowledge-Based Systems], Springer-Verlag, New York City, New York, (1991), update 2011. 
[61] Mahler, R. P. S., "Random Sets in Information Fusion," in Random Sets: Theory and Applications, Eds. J. Goutsias, R.P.S. Mahler, H.T. Nguyen, IMA Volumes in Mathematics and its Applications, Vol. 97, Springer-Verlag Inc., New York, pp. 129-164, (1997).

[62] Hestir, K., Nguyen, H.T., Rogers, G.S., “A random set formalism for evidential reasoning," in Conditional Logic in Expert Systems (I.R. Goodman, N.M. Gupta, H.T. Nguyen, and G.S. Rogers, eds.) Amsterdam, The Netherlands: North-Holland, (1991).

[63] Nguyen, H. T., "On random sets and belief function," J. of mathematical Analysis and Applications, Vol 65, pp. 531-542, (1978).

[64] Smets, P., "The transferable belief model and random sets," International J. of Intelligent Systems, Vol 7, pp. 37 - 46, (1992).

[65] Dezert, J. "Non-Bayesian Reasoning for Information Fusion - A Tribute to Lofti Zadeh," submitted to J. of Adv. of Information Fusion, (2012).

[66] Yen, J., "A reasoning model based on the extended Dempster Shafer theory," Nat Conf. on Artificial Intelligence, (1986).

[67] Mahler, R.P., "Classification when a priori evidence is ambiguous," Proc. SPIE, Vol. 2234, (1994).

[68] Mahler, R.P., "Combining ambiguous evidence with respect to ambiguous a priori knowledge, I: Boolean logic," IEEE Trans. Sys., Man \& Cyber., Part A, Vol. 26, pp. 27-41, (1996).

[69] Hong, L., Lynch, A, "Recursive temporal-spatial information fusion with applications to target identification," IEEE Transactions on Aerospace and Electronic Systems, Volume: 29 , Issue: 2, pp 435 - 445, (1993).

[70] Buede, D. M., Girardi, P., “A target identification comparison of Bayesian and Dempster-Shafer multisensor fusion," IEEE Transactions on Systems, Man and Cybernetics, Part A: Systems and Humans, Volume: 27 , Issue: 5 , pp. 569 - 577, (1997).

[71] Blasch, E., "Learning attributes for situational awareness in the landing of an autonomous airplane," AIAA/IEEE Digital Avionics Systems Conference, (1997).

[72] Leung, H., Wu, J., "Bayesian and Dempster-Shafer target identification for radar surveillance," IEEE Transactions on Aerospace and Electronic Systems, Volume: 36 , Issue: 2, pp. 432 - 447, (2000).

[73] Schuck, T.M., Shoemaker, B., Willey, J., "Identification friend-or-foe (IFF) sensor uncertainties, ambiguities, deception and their application to the multi-source fusion process," IEEE National Aerospace and Electronics Conference, (2000).

[74] Mahler, R., "Can the Bayesian and Dempster-Shafer approaches be reconciled? Yes," Int'l Conf. on Information Fusion, (2005).

[75] Maskell, S. "A Bayesian approach to fusing uncertain, imprecise and conflicting information," Information Fusion, Vol. 9, 2, pp. 259277, April (2008).

[76] Dezert, J. Smarandache, F., [Advances and applications of DSmT for information fusion (Collected works)], Vols. 1-3, American Research Press, 2004-2009. http://fs.gallup.unm.edu/DSmT.htm

[77] Dezert, J. "Foundations for a new theory of plausible and paradoxical reasoning," Information \& Security, An Int'l J., ed. by Prof. Tzv. Semerdjiev, Vol. 9, (2002).

[78] Dezert, J. Smarandache, F., "On the generation of hyper-powersets for the DSmT," Int. Conf. on Info Fusion, (2003).

[79] Shafer, G., [A Mathematical Theory of Evidence], Princeton, NJ: Princeton Univ. Press, (1976).

[80] Smets, P., "Analyzing the Combination of Conflicting Belief Functions," (2005).

[81] Daniel, M., "The MinC Combination of Belief Functions, derivation and formulas," Tech Report No. 964, Acad. Sci. of the Czech Republic, (2006).

[82] Jøsang, A., Daniel, M., “Strategies for Combining Conflict Dogmatic Beliefs," Int. Conf. on Info Fusion, (2006).

[83] Daniel, M., "Generalization of the Classic Combination Rules to DSm Hyper-Power Sets," Information \& Security, An Int'l J., Vol. 20, (2006).

[84] Smaradache, F., Dezert, J., "Information fusion based on new proportional conflict redistribution rules," Int. Conf. Inf. Fusion, (2005).

[85] Florea, M. C., Dezert, J., Valin, P., Smarandache, F., Jousselme, A-L., "Adaptive combination rule and proportional conflict redistribution rule for information fusion," COGIS '06 Conf., (2006).

[86] Martin, A., Osswald, C., Dezert, J., Smarandache, F. “General Combination Rules for Qualitative and Quantitative Beliefs,” J. of Advances in Information Fusion, Vol. 3, No. 2, Dec. (2008).

[87] Valin, P., Djiknavorian, P, Bossé, E., "A Pragmatic Approach for the use of Dempster-Shafer Theory in Fusing realistic Sensor Data," J. of Advances in Info. Fusion, Vol. 5, No. 1, June (2010).

[88] Djiknavorian, P., Grenier, D., Valin, P. "Approximation in DSm theory for fusing ESM reports," Int. Workshop on Belief functions 2010, Brest, France, April (2010).

[89] Lee, Z. H., Choir, J. S., Elmasri, R., "A Static Evidential Network for Context Reasoning in Home-Based Care," IEEE Trans. Sys., Man, and Cyber-Part A; Sys \& Humans, Vol. 40, No. 6, Nov, (2010).

[90] Blasch, E., Dezert, J., Valin, P., "DSMT Applied to Seismic and Acoustic Sensor Fusion," Proc. IEEE Nat. Aerospace Electronics Conf (NAECON), (2011).

[91] Martin, A., Osswald, C., "Generalized Proportion conflict redistribution rule applied to sonar imagery and radar targets classification," in Adv. and Appl. of DSmT for Information Fusion, Vol. 2, (Eds.) J. Dezert and F. Smarandache, (2006).

[92] Smarandache, F., Dezert, J., "On the consistency of PCR6 with the averaging rule and its application to probability estimation," Int. Conf. on Info Fusion, (2013).

[93] Dezert, J., Smarandache, F., “A new probabilistic transformation of belief mass assignment,” Int. Conf. on Info Fusion, (2008).

[94] Yang, C., Kaplan, L., Blasch, E., "Performance Measures of Covariance and Information Matrices in Resource Management for Target State Estimation," IEEE Transactions on Aerospace and Electronic Systems, Vol. 48, No. 3, pp. 2594 - 2613, (2012).

[95] http://mmsip.bas.bg/mmosi/partners/jeandezert.html

[96] Mei, X., Ling, H., Wu, Y., Blasch, E., Bai, L. "Efficient Minimum Error Bounded Particle Resampling L1 Tracker with Occlusion Detection," IEEE Trans. on Image Processing (T-IP), (2013). 